\title{
Vancomycin-resistant enterococci (VRE) screening and isolation in the general medicine ward: a cost-effectiveness analysis
}

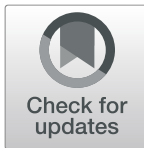

Stephen Mac $^{1,2^{*}}$ (D), Tiffany Fitzpatrick ${ }^{3}$, Jennie Johnstone ${ }^{3,4,5}$ and Beate Sander ${ }^{1,2,5,6}$

\begin{abstract}
Background: Vancomycin-resistant enterococci (VRE) are a serious antimicrobial resistant threat in the healthcare setting. We assessed the cost-effectiveness of VRE screening and isolation for patients at high-risk for colonisation on a general medicine ward compared to no VRE screening and isolation from the healthcare payer perspective.

Methods: We developed a microsimulation model using local data and VRE literature, to simulate a 20-bed general medicine ward at a tertiary-care hospital with up to 1000 admissions, approximating 1 year. Primary outcomes were accrued over the patient's lifetime, discounted at 1.5\%, and included expected health outcomes (VRE colonisations, VRE infections, VRE-related bacteremia, and deaths subsequent to VRE infection), quality-adjusted life years (QALYs), healthcare costs, and incremental cost-effectiveness ratio (ICER). Probabilistic sensitivity analysis (PSA) and scenario analyses were conducted to assess parameter uncertainty.
\end{abstract}

Results: In our base-case analysis, VRE screening and isolation prevented six healthcare-associated VRE colonisations per 1000 admissions (6/1000), 0.6/1000 VRE-related infections, 0.2/1000 VRE-related bacteremia, and 0.1/1000 deaths subsequent to VRE infection. VRE screening and isolation accrued 0.0142 incremental QALYs at an incremental cost of $\$ 112$, affording an ICER of $\$ 7850$ per QALY. VRE screening and isolation practice was more likely to be costeffective (> 50\%) at a cost-effectiveness threshold of $\$ 50,000 / Q A L Y$. Stochasticity (randomness) had a significant impact on the cost-effectiveness.

Conclusion: VRE screening and isolation can be cost-effective in majority of model simulations at commonly used cost-effectiveness thresholds, and is likely economically attractive in general medicine settings. Our findings strengthen the understanding of VRE prevention strategies and are of importance to hospital program planners and infection prevention and control.

Keywords: Infection control, Vancomycin-resistant enterococci, VRE, Hospital-acquired infection, Antimicrobial resistance, Health economics, Cost-effectiveness analysis

\section{Introduction}

Vancomycin-resistant enterococci (VRE) are a class of antimicrobial resistant (AMR) bacteria most commonly transmitted within healthcare settings [1]. While immunocompetent patients have a low risk of acquiring VRE infections post-colonisation, other patient groups

\footnotetext{
* Correspondence: sm.mac@mail.utoronto.ca

${ }^{1}$ Institute of Health Policy, Management and Evaluation, University of Toronto, 155 College Street, Suite 425, Toronto, ON M5T 3M6, Canada ${ }^{2}$ Toronto Health Economics and Technology Assessment (THETA) Collaborative, University Health Network, 200 Elizabeth Street, 10th Floor, Room 247, Toronto, ON M5G 2C4, Canada

Full list of author information is available at the end of the article
}

(e.g. immunocompromised, oncology, transplant) are at a higher risk of developing VRE-related bacteremia and other infections [2]. Consequently, patients who develop VRE-related infections require longer hospital stays, have a higher risk of mortality, and substantially higher medical costs. A study from Canada estimated the mean attributable cost and length of stay for patients with VRE colonisation/infection to be $\$ 17,949$ and 13.8 days, respectively, when compared to patients without VRE [3].

Guidelines for control of VRE from health agencies (e.g. Centers of Disease Control and Prevention) in the United States and the United Kingdom recommend

(c) The Author(s). 2019 Open Access This article is distributed under the terms of the Creative Commons Attribution 4.0 International License (http://creativecommons.org/licenses/by/4.0/), which permits unrestricted use, distribution, and 
control of VRE spread through vancomycin usage, screening and isolation of patients with VRE in hospital settings, education, cleaning and contact precautions (e.g. gloves) $[4,5]$. Similarly in Canada, provincial committees recommend the implementation of active VRE screening programs for patients at high-risk of VRE colonisation [6]. Risk factors for VRE colonisation include: previous admission to healthcare facilities (e.g. hospital); dialysis recipient; transfer from long-term care facilities; and previous receipt of certain classes of antibiotics (e.g. cephalosporin) [6].

In 2014, the Canadian Agency for Drugs and Technology for Health (CADTH) conducted a rapid response review on the cost-effectiveness of patient screening and isolation for VRE and identified one economic evaluation from France, where the direct cost of an outbreak triggered by a failure in systematic VRE screening had a direct cost of $€ 60,524$ [7]. Two economic evaluations from hospital settings reported a net benefit of using a VRE control strategy $[8,9]$.

Based on the current literature, there are no costeffectiveness analyses for VRE screening and isolation practices that included health outcomes in evaluating the value of this control strategy. The objective of our study was to conduct a cost-effectiveness analysis of active VRE screening and isolation compared to no VRE screening and isolation in the general medicine ward of a tertiary care hospital. Due to conflicting evidence on the value of prevention programs for VRE, we decided to model a general medicine ward instead of an intensive care unit (ICU) because of its heterogeneous nature (i.e. varying patient risk for VRE colonisation and infections). Evidence from this model can inform decisionmakers, program planners and clinicians contemplating control strategies for healthcare-associated VRE-related infections.

\section{Methods}

A cost-effectiveness analysis (CEA) was conducted from the Ontario healthcare payer perspective (Ministry of Health and Long-Term Care). Health outcomes were accrued over a patients' lifetime and included: healthcare-associated VRE colonisations, VRE-related infections (e.g. bacteremia and other infections), deaths subsequent to VRE infection, and quality-adjusted life years (QALY). All publicly-funded healthcare costs (2017 Canadian dollars) were included. The primary outcomes were total healthcare costs, QALYs, and the incremental cost-effectiveness ratio (ICER) expressed in $\$$ per QALY gained. Cost-effectiveness of VRE screening and isolation was assessed against the commonly used cost-effectiveness threshold (CET) of $\$ 50,000$ per QALY gained [10]. We followed CADTH guidelines and reported outcomes discounted at 1.5\% [11].

\section{Model structure and patient population}

A microsimulation model was developed to capture the natural history of VRE health burden starting at hospital admission. Schematics of the model are presented in Figs. 1, 2 and 3. The model simulated a dynamic population of 20 patients in the general medicine ward, i.e., patient flow was simulated by admitting a new patient to the ward once an existing patient was discharged back into the community, or died during their hospital stay. Admitted patients were considered to be from the community; we did not take into account entry from long-term care facilities, readmissions, or ICU step-downs. For basecase analysis, we evaluated the cost-effectiveness of VRE screening and isolation through 1000 admissions, approximating 1 year. After 1000 admissions, hospital admissions stopped, and patients were followed over their lifetime. All modelling and analyses were conducted using TreeAge Pro 2018 (TreeAge Software, Inc., Williamstown, MA).

\section{VRE transmission}

A two-state dynamic transmission component simulated VRE transmission. The probability of acquiring VRE responds to changes to the number of VRE-colonised patients in the ward who are not isolated and was modeled using the following equation [12]:

$$
\mathrm{C}_{\mathrm{t}+1} / \mathrm{S}_{\mathrm{t}}=1-e^{-\beta C t / N}
$$

Where $t$ represents the specific cycle or time period, $\mathrm{C}_{\mathrm{t}+1}$ is the number of patients who are VRE colonised (but not isolated) in the current cycle, $\mathrm{N}$ is total number of patients, $S_{t}$ represents the total number of patients susceptible to VRE colonisation in the previous cycle, and $\beta$ is the basic reproductive number of VRE. The basic reproductive number was defined as the number of new infections generated per infected (non-isolated) individual per unit of time. For our model, we assumed a constant basic reproductive number of 1.32 .

\section{Key assumptions}

Several key assumptions were made on VRE transmission and isolation parameters. These included: 1) VRE rectal swab screen are completed concurrently with Methicillin-resistant Staphylococcus aureus (MRSA) rectal swab screening (i.e., only additional cost is processing the swab), and results are delivered within $24 \mathrm{~h}$, a period in which colonized patients can contribute to transmission; 2) transmission is based solely on mass-action mixing; 3) optimal adherence to isolation (i.e. isolation is $100 \%$ effective in reducing transmission); 4) cost of private (single-bed) room, which is typically considered hospital revenue, is captured in the healthcare payer perspective; 5) the general medicine ward has 20 single-bed rooms, always at maximum capacity; and 6) colonization 

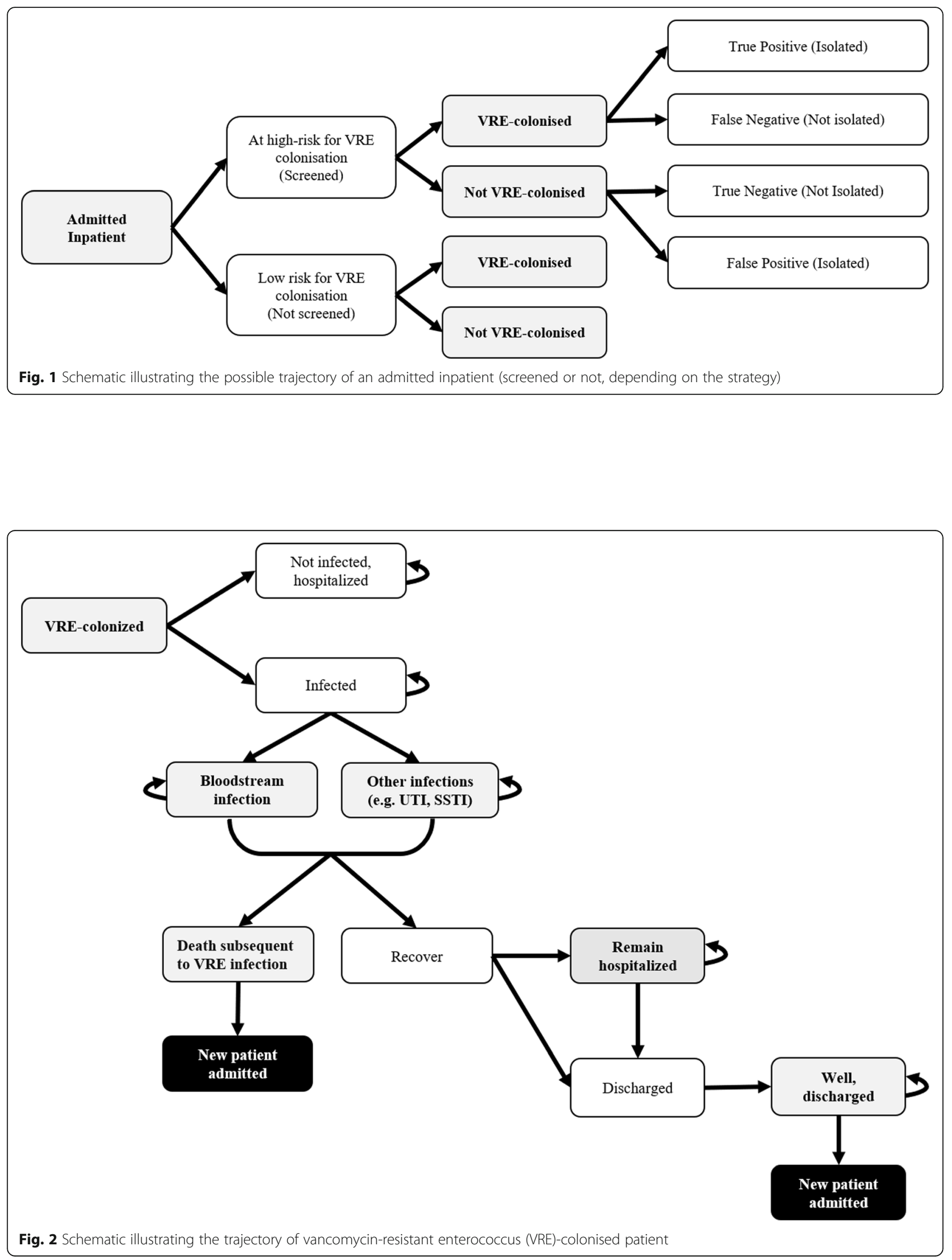


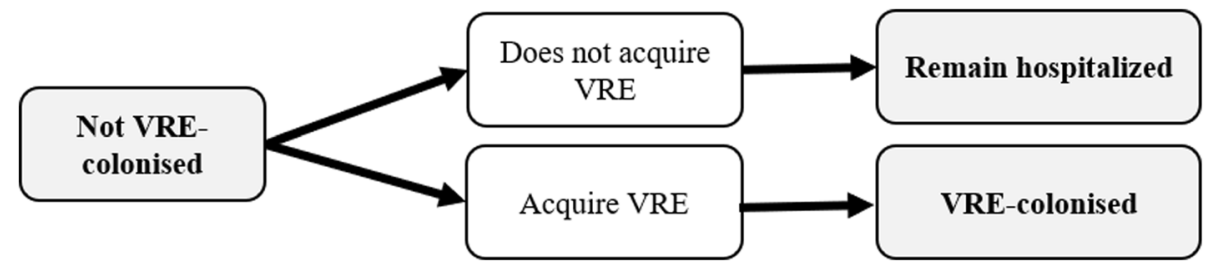

Fig. 3 Schematic illustrating the possible trajectory of patient not VRE-colonised

status of the prior patient in the room was not factored into transmission.

\section{Data sources}

A targeted literature search was conducted to extract outcome probabilities, costs and quality-of-life parameters related to VRE health states (Table 1). When possible, Canadian-specific parameters were used. Where "assumption" is indicated in Table 1, we were guided by expert opinion.

\section{Probabilities}

The basic reproductive rate for VRE was uncertain and can vary depending on the environment. We used results from a meta-analysis of 10 studies that reported a reproductive rate of 1.32 (95\% CI, 1.03-1.46) [13]. Length of stay (LOS) estimates used for patients with VRE infections was 39 days (IQR, 22-81 days) and without VRE infections was 3 days (IQR, 1-6 days), extracted from a case-control study in Canada [2]. We used a screening rectal swab sensitivity of 0.99 (95\% CI, 0.952-1.00) and specificity of 0.948 (95\% CI, 0.922-0.968) from an United States study evaluating the swab detection of E. faecium and E. faecalis [16]. Prevalence of VRE for low-risk patients was 0.023, which was extracted from a Canadian study in 2012 [14]. The probability that a patient was at "high-risk" of colonisation was guided by the average age (61 years) of the cohort of patients who acquired VRE-bacteremia in Canada [2]. Allcause mortality from all-causes were derived from life tables from Statistics Canada [29].

\section{Utilities}

To properly value health outcomes for CEAs, we used health state utility values (utilities), which is a preferencebased value expressing the quality-of-life associated with health states [30]. Utilities for this study could have ranged between 0 (health state equivalent to death) to one (perfect health). The utility of a VRE-colonised patient was considered to be the same as that of a general inpatient (0.642), which was obtained from a mixed population of inpatients using the EuroQol rating scale [25]. The utility for the well outpatient state was derived from a study of community-dwelling adults using the Health Utilities Index to be 0.93 ( 0.86 for patients who recovered from a
VRE-related infection) [28]. Due to data limitations, bacteremia utility (0.56) was extracted from a MRSArelated bacteremia study [23]. Since urinary tract infections (UTI) represented the greatest percentage of VRErelated infections $[3,31]$, we used the UTI utility of 0.60 for all other infections [24]. We assumed a disutility with being isolated (i.e. being isolated leads to less visits from healthcare workers, reduced socialization, and space confinement), which was equivalent to mild depression (untreated), and applied a multiplicative 0.895 reduction factor [27].

\section{Costs}

All direct costs were extracted from the literature (Table 1 ). We counted the cost of the screening as a one-time upfront cost at ward admission between $\$ 12$ and $\$ 24$, depending on the culture result (positive results being more expensive due to additional microbiologist time required) [9]. All costs were converted and standardized to 2017 Canadian dollars. For private room costs, we used the median from estimates across Ontario (\$290 per night) [21].

\section{Analysis}

The base-case analysis was defined as follows: screening with $95 \%$ specificity and $99 \%$ sensitivity, VRE basic reproductive number of 1.32 [13], and mean age of highrisk patients at 61 years [2]. The baseline prevalence of VRE was 0.023 and we assumed patients at higher risk for VRE colonisation were four times more likely to be colonised (0.092). The base-case analysis was conducted from a Canadian perspective.

We conducted multiple scenario analysis including: universal screening and isolation for all patients, increased duration of the program (5000 admissions), number of beds, and a lower effectiveness (compliance) of the isolation program.

We conducted a probabilistic sensitivity analysis (PSA) using gamma distributions for costs, beta distributions for utilities and transitional probabilities, and normal distributions for other patient or VRE-related parameters (see Table 1). From the PSA, we generated a costeffectiveness acceptability curve (CEAC) to determine the probability of VRE screening and isolation being 
Table 1 Input parameter base-case values, plausible ranges and distributions

\begin{tabular}{|c|c|c|c|c|c|c|}
\hline Variable & Base-case value & Range & Range Type & Standard Error & Distribution & Source \\
\hline \multicolumn{7}{|l|}{ VRE-Related Parameters } \\
\hline Beta, basic reproductive number & 1.32 & $1.03-1.46$ & Full & 0.12 & Gamma & Satilmis 2016 [13] \\
\hline VRE prevalence, general & 0.023 & $0-0.18$ & Full & 0.001 & Beta & Williams 2015 [14] \\
\hline VRE prevalence, high-risk patients & 0.092 & $0-0.36$ & Plausible & 0.002 & Beta & Conly 2001 [15] \\
\hline LOS | without VRE infection, days & 3 & $1.0-6.0$ & Full (IQR) & 0.38 & Gamma & Johnstone 2018 [2] \\
\hline LOS | other VRE infection, days & 6 & $1.0-6.0$ & Full (IQR) & 0.77 & Gamma & $\begin{array}{l}\text { Assumption; } \\
\text { Johnstone } 2018 \text { [2] }\end{array}$ \\
\hline LOS | VRE-bacteremia, days & 39 & $22.0-81.0$ & Full (IQR) & 4.97 & Gamma & Johnstone 2018 [2] \\
\hline \multicolumn{7}{|l|}{ Screening Parameters } \\
\hline Sensitivity, rectal swab & 0.991 & $0.95-1.00$ & Full & 0.02 & Beta & Stamper 2010 [16] \\
\hline Specificity, rectal swab & 0.949 & $0.92-0.97$ & Full & 0.01 & Beta & Stamper 2010 [16] \\
\hline Effectiveness of isolation & 1.00 & $0.75-1.00$ & Plausible & - & & Assumption \\
\hline Discount rate, annual & 0.015 & $0-0.03$ & Full & - & - & CADTH 2017 [11] \\
\hline \multicolumn{7}{|l|}{ Patient Parameters and Transition Probabilities } \\
\hline Average age high-risk, years & 61 & - & - & 1.15 & Normal & Johnstone 2018 [2] \\
\hline Probability infected | colonised & 0.025 & $0.018-0.031$ & Plausible & 0.003 & Beta & Williams 2015 [14] \\
\hline Probability bacteremia | infected & 0.155 & $0.12-0.19$ & Plausible & 0.02 & Beta & Saunders 2004 [17] \\
\hline Odds ratio bacteremia | infected, high-risk & 1.55 & $0.56-4.29$ & Full & 1.68 & Lognormal & Johnstone 2018 [2] \\
\hline Average days of treatment for BSI & 14 & $11-18$ & Plausible & 1.79 & Gamma & Daneman 2016 [18] \\
\hline Average days of treatment for other infections & 7 & $5-9$ & Plausible & 0.89 & Gamma & Daneman 2016 [18] \\
\hline $\begin{array}{l}\text { Probability of death from VRE bacteremia, } \\
\text { average, } 14 \text { days }\end{array}$ & 0.37 & $0.27-0.46$ & Plausible & 0.05 & Beta & Billington 2014 [19] \\
\hline $\begin{array}{l}\text { Probability of death from VRE bacteremia, } \\
\text { high-risk, } 14 \text { days }\end{array}$ & 0.46 & $0.35-0.58$ & Plausible & 0.06 & Beta & Linden 1996 [20] \\
\hline Number of room visits by all HCW, per day & 24 & $18-30$ & Plausible & 3.06 & Normal & Assumption \\
\hline \multicolumn{7}{|l|}{ Costs } \\
\hline Rectal swab screen & 3.13 & $2.35-3.91$ & Plausible & 0.40 & Gamma & Muto 2002 [9] \\
\hline Culture, positive test & 21.36 & $16.02-26.7$ & Plausible & 2.72 & Gamma & Muto 2002 [9] \\
\hline Culture, negative test & 8.97 & $6.73-11.21$ & Plausible & 1.14 & Gamma & Muto 2002 [9] \\
\hline PPE, per room visit & 2.10 & $1.58-2.63$ & Plausible & 0.27 & Gamma & Muto 2002 [9] \\
\hline Nurse time, per test & 7.12 & $5.34-8.9$ & Plausible & 0.91 & Gamma & Muto 2002 [9] \\
\hline Private room, daily & 290 & $245-410$ & Full & - & - & $\begin{array}{l}\text { St. Joseph's } \\
\text { Hospital } 2017 \text { [21] }\end{array}$ \\
\hline Antibiotics, bacteremia, daily & 524.22 & $393.17-655.28$ & Plausible & 66.87 & Gamma & Nasr 2011 [22] \\
\hline Antibiotics, other infections, daily & 35.8 & $26.85-44.75$ & Plausible & 4.57 & Gamma & Nasr 2011 [22] \\
\hline \multicolumn{7}{|l|}{ Utilities } \\
\hline VRE bacteremia & 0.56 & $0.51-0.61$ & Full & 0.023 & Beta & Lee 2010 [23] \\
\hline Other local infections (UTI) & 0.60 & $0.58-0.62$ & Full & 0.01 & Beta & Haran 2005 [24] \\
\hline Inpatient & 0.642 & $0.54-0.74$ & Full & 0.05 & Beta & $\begin{array}{l}\text { Tengs, } 2000[25] ; \\
\text { Selai } 1995[26]\end{array}$ \\
\hline Mild depression, no treatment & 0.88 & $0.84-0.92$ & Full & 0.02 & Beta & Revicki 1997 [27] \\
\hline $\begin{array}{l}\text { Well, chronic conditions, recovered from } \\
\text { previous VRE-related infection }\end{array}$ & 0.86 & $0.34-0.89$ & Full & 0.15 & Beta & Mittmann 1999 [28] \\
\hline $\begin{array}{l}\text { Well, chronic conditions, no previous } \\
\text { VRE-related infection }\end{array}$ & 0.93 & $0.88-0.94$ & Full & 0.083 & Beta & Mittmann 1999 [28] \\
\hline
\end{tabular}


cost-effective at CET of $\$ 0$ to $\$ 100,000$ per QALY. We also assessed expected value of perfect information at several CETs to assess the value of information; i.e., whether or not to invest more resources to reduce parameter uncertainty. As recommended by CADTH, we did not conduct deterministic sensitivity analysis because of model stochasticity and the non-linear relationship of VRE prevalence and transmission parameters. We reported results following the Consolidated Health Economic Evaluation Reporting Standards (CHEERS) Guidelines (Additional file 1) [32].

\section{Results}

\section{Base-case analysis}

In Table 2, we summarized the estimated health outcomes, costs and ICER for the VRE screening and isolation strategy compared to no VRE screening and isolation over 1000 admissions for our base-case analysis. We calculated the difference in the health outcomes and the relative change using the "no VRE screening and isolation" strategy as the baseline. VRE screening and isolation reduced healthcare-associated VRE colonisations by six per 1000 patients $(2 / 1000$ with screening and isolation vs. $8 / 1000$ without, $73 \%$ reduction), VRE-related infections by 0.6 per 1000 patients (5.7/1000 with screening and isolation vs. 6.3/1000 without, $10 \%)$, VRE-related bacteremia by 0.2 per 1000 patients $(2.5 / 1000$ with screening and isolation vs. $2.7 / 1000$ without, $7 \%$ ) and deaths subsequent to VRE infection by 0.1 per $1000(0.5 / 1000$ with screening and isolation vs. $0.6 / 1000$ without, $8 \%$ ).

The incremental cost and effect for VRE screening and isolation was $\$ 110$ ( $\$ 118.37$ with screening and isolation vs. $\$ 6.72$ without), and 0.0142 QALY gained (20.5607 QALY with screening and isolation vs. 20.5465 QALY without), respectively. The ICER for VRE screening and isolation was $\$ 7850$ per QALY gained.

\section{Uncertainty: probabilistic sensitivity analysis}

Figure 4 illustrates a CEAC where at low CETs below $\$ 7500 /$ QALY, it was unlikely that VRE screening and isolation was a cost-effective strategy. At a CET of approximately $\$ 7500 / \mathrm{QALY}, \mathrm{VRE}$ screening and isolation became more likely to be cost-effective (over $50 \%$ of the iterations). As the CET increased to $\$ 50,000$ per QALY, the probability of this program being cost-effective asymptotes at approximately $51.4 \%$.

Since VRE screening and isolation reached a plateau of $51 \%$ likelihood of being cost-effective, an expected value of perfect information (EVPI) analysis was conducted to determine the value of reducing further uncertainty at three points. At a CET of $\$ 7500$, and $\$ 50,000$ per QALY, the EVPI (assuming 1000 patients) was $\$ 1065$, and $\$ 7093$, respectively.

\section{Scenario analysis}

In the scenario where the prevalence is lower (i.e. reduced by half; 0.0115), VRE screening and isolation becomes a dominated strategy: the program cost an additional $\$ 123$ but resulted in fewer QALYs. On the other hand, we modeled a scenario similar to outbreaks in the literature where the VRE prevalence was about 10-fold higher (0.23), and estimated that VRE screening and isolation cost $\$ 122.79$ for an incremental increase of 0.0525 QALY. Under this increased prevalence scenario over 1000 hospital admissions, the ICER was \$2340/QALY. All scenarios are summarized in Table 3.

Scenario analysis was conducted where the private room costs were excluded due to conflicting views on whether these costs are considered from the Ontario healthcare payer perspective. In this scenario, VRE screening and isolation program cost an additional $\$ 20.58$ for 0.0077 QALYs, resulting in an ICER of \$2682/QALY. The number of beds in the simulated general medicine ward was increased to 30. The cost-effectiveness of VRE screening and isolation over 5000 admissions was also estimated.

Table 2 Base-case results (health and economic outcomes)

\begin{tabular}{llll}
\hline Outcomes & VRE screening and isolation & No VRE screening and isolation & Difference $^{\text {a }}(\%)$ \\
\hline Non-isolated cases & $11 / 1000$ & $60 / 1000$ & $-49 / 1000(82 \%)$ \\
Healthcare-associated VRE-colonisation & $2 / 1000$ & $8 / 1000$ & $-6 / 1000(73 \%)$ \\
Infected cases & $5.7 / 1000$ & $6.3 / 1000$ & $-0.6 / 1000(10 \%)$ \\
VRE-related bacteremia & $2.6 / 1000$ & $2.8 / 1000$ & $-0.2 / 1000(7 \%)$ \\
Other VRE infections (e.g. UTI) & $3.2 / 1000$ & $3.6 / 1000$ & $-0.4 / 1000(12 \%)$ \\
Deaths subsequent to VRE infection & $0.5 / 1000$ & $0.6 / 1000$ & $-0.1 / 1000(8 \%)$ \\
ICER (\$/QALY) & & & 7850 \\
$\quad$ Total costs (\$) & 118.37 & 6.72 & 112 \\
$\quad$ Total QALY gained & 20.5607 & 20.5465 & 0.0142 \\
\hline
\end{tabular}

"Difference for health outcomes were calculated by subtracting "no VRE screening and isolation strategy" outcomes from "VRE screening and isolation strategy" outcomes. Percentage change was calculated relative to "no VRE screening and isolation strategy" outcomes

ICER incremental cost-effectiveness ration, QALY quality-adjusted life years, UTI urinary tract infection, VRE vancomycin-resistant enterococci 


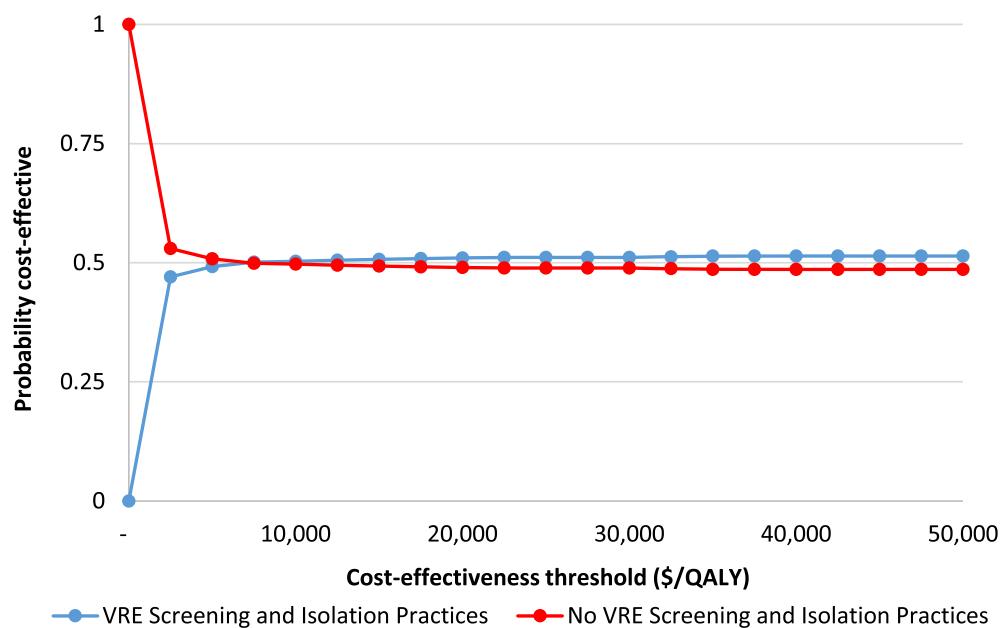

Fig. 4 Cost-effectiveness acceptability curve (CEAC) for cost-effectiveness thresholds from $\$ 0$ to $\$ 50,000 /$ Q $A L Y$

The estimated ICERs for these scenarios were $\$ 11,812$ / QALY and \$50,094/QALY, respectively.

Universal VRE screening and isolation for all patients, regardless of whether they identified as high-risk for colonisation, was a dominated strategy (i.e. resulted in incremental cost of $\$ 151.44$ and QALYs lost). We also estimated the cost-effectiveness of this program if the isolation effectiveness was reduced to $75 \%$. In this scenario, VRE screening and isolation cost an additional $\$ 99.52$ for 0.0002 QALYs, resulting in an ICER of $\$ 510$, 676/QALY.

\section{Discussion}

Based on our base-case analysis, VRE screening and isolation for patients at high-risk for VRE colonisation prevented healthcare-associated colonisations, and ultimately VRE-related infections and deaths subsequent to infections. The program was considered cost-effective with an
ICER of $\$ 7850$ per QALY when compared to commonly used cost-effectiveness thresholds of \$50,000/QALY [10].

Overall, our model's results were consistent with the findings of several other published studies [34-37]. A study by Shadel et al. found that active VRE screening and isolation resulted in 91\% of VRE colonisations being identified on an ICU; our model suggested $82 \%$ of VRE positive patients were isolated under an active, targeted screening strategy in a general medicine ward [34]. A mathematical model of a 10-bed ICU active screening program for VRE predicted 9.9 cases of VRE colonisation/infection prevented over 1000 model simulations in the ICU with a prevalence rate of 5\% [35]. Similarly, our model predicts a reduction of 6 cases of VRE colonisations over 1000 admissions. Our model underestimated the effect of the VRE screening and isolation compared to both studies, likely because it was modeled after a general medicine ward which has a lower proportion of high-risk patients (for VRE colonisation and infection) than the

Table 3 Incremental cost-effectiveness ratios for VRE screening and isolation program in various scenarios

\begin{tabular}{llllll}
\hline Scenario & Incremental Cost & Incremental QALYS & ICER (\$/QALY) & $\begin{array}{l}\text { Probability of CE } \\
\text { (at \$7500/QALY) }\end{array}$ & $\begin{array}{l}\text { Probability of CE } \\
\text { (at \$50,000/QALY) }\end{array}$ \\
\hline VRE Prevalence in-hospital, 10x (outbreak) & 122.79 & 0.0525 & 2340 & 0.545 & $0.556^{\mathrm{a}}$ \\
Room costs excluded (\$0) & 20.58 & 0.0077 & 2682 & 0.506 & $0.508^{\mathrm{a}}$ \\
Number of beds in ward [33] & 109.78 & 0.0093 & 11,812 & 0.505 & $0.518^{\mathrm{a}}$ \\
Program length (5000 admissions) & 113.05 & 0.0023 & 50,094 & 0.457 & $0.499^{\mathrm{a}}$ \\
Isolation, decreased effectiveness (0.75) & 99.52 & 0.0002 & 510,676 & 0.458 & $0.476^{\mathrm{a}}$ \\
Time horizon, 1 year & 109.61 & 0.0001 & 856,297 & 0 & 0.259 \\
Universal screening VRE screening & 151.44 & -0.0039 & Dominated & 0.484 & $0.500^{\mathrm{a}}$ \\
and isolation & & & & 0.479 \\
VRE Prevalence in-hospital, 0.5x & 108.41 & -0.0112 & Dominated & & $0.501^{\mathrm{a}}$ \\
\hline
\end{tabular}

${ }^{\text {a Signifies asymptote at that probability at } \$ 50,000 / \mathrm{QALY}}$

$C E$ cost-effectiveness, ICER incremental cost-effectiveness ratio, $Q A L Y$ quality-adjusted life year, VRE vancomycin-resistant enterococci 
ICU. However, similar to other studies, our model estimated that active VRE screening and isolation strategy was cost-effective by reducing the number of VRE-related bacteremia events by $2 / 10,000$ patients $[36,37]$.

Our study has several limitations. Health state utilities were not specific to VRE infections and preference elicitation was heterogeneous. To address this and other parameter uncertainty (e.g. costs and transition probabilities), we conducted a PSA with the appropriate underlying distribution for all parameters to generate a CEAC for costeffectiveness thresholds of $\$ 0$ to $\$ 50,000 / Q A L Y$. VRE screening and isolation was more likely to be cost-effective than no VRE screening and isolation at a CET of $\$ 7500$ / QALY or greater. However, as the CET increased to $\$ 50$, 000/QALY, the likelihood of the program being costeffective in extended simulations remained steady at $51 \%$, suggesting that stochasticity (randomness) is a significant factor in determining the value of this control program. This was expected for this type of intervention since individual level uncertainty with patients entering a general medicine ward and the baseline VRE prevalence can influence VRE transmission.

Our study assumed a general medicine ward that was set up with 20 single bed rooms, which may not be the configuration of all general medicine wards. In a scenario where 30-beds were used, the ICER increased to $\$ 11,812 / \mathrm{QALY}$. These results suggest that an increase in the number of beds would still yield cost-effective VRE screening and isolation practices due to the homogeneous mixing assumption. This assumption was made despite knowing that VRE transmission can be highly complex and depend on colonization pressure and density of bacteria [33]. Incorporating such detail of VRE colonization levels within the transmission modeling of this CEA would require much more sophisticated VRE surveillance data that was not available. We did not explore the value of this program in which patients shared rooms. However, based on Hamel et al., the hazard ratio for VRE colonisation was 1.11 (95\% CI, 1.02-1.21) for the number of roommate exposures per day [38]. Our estimates using a single-bed room assumption was a conservative approach, and therefore likely underestimated the cost-effectiveness of a VRE screening and isolation control program. Our model likely provided a conservative estimate of the cost-effectiveness (i.e. underestimates the value) of VRE screening and isolation due to key assumptions required for our analysis (e.g. did not incorporate time dependency within the ward, or re-admissions).

Isolation was assumed to be completely effective in our base-case analysis, which can be considered optimistic in current healthcare settings given the potential for human errors, and overall burden on healthcare workers $[39,40]$. We performed a scenario analysis based on a study by Huskins and colleagues suggesting prevention effectiveness of $75 \%$ (range 62-82\%) [40], and the ICER increased to $\$ 510,676 /$ QALY. In this scenario, VRE screening and isolation would unlikely be cost-effective at commonly used thresholds. Due to stochasticity (randomness), it is likely that the cost-effectiveness and isolation effectiveness have a nonlinear relationship. This may be of note to decision-makers and infection prevention and control practitioners, to ensure implementation of this program is as seamless as possible.

Cost-effectiveness analyses for screening programs of other AMR bacteria such as carbapenemase-producing Enterobacteriaceae and MRSA have been published in the literature $[41,42]$. Similar to these other economic evaluations on AMR bacteria screening and isolation, our results indicated that VRE screening and isolation was likely to be cost-effective. To our knowledge, this is the first cost-effectiveness analysis for VRE screening and isolation in any hospital setting that incorporated costs, health outcomes, and QALYs, accrued over a patient's lifetime. We reported health outcomes per 1000 patients to allow for transferability of our results to general medicine wards in different jurisdictions. Moreover, the results of this cost-effectiveness analysis can be generalizable to other jurisdictions (countries) with similar healthcare system financing to Canada such as Australia, the United Kingdom, and parts of Europe. We also estimated the cost-effectiveness of this program in varying scenarios (e.g. varying VRE prevalence, number of beds) to provide decision-makers with economic evidence to support local health policy given the importance of local context.

Given the limited body of evidence in this area, we were unable to find a suitable source of data against which to validate our results. As more local research on AMR bacteria continues, it will allow for future models to be crossvalidated to health outcomes using health administrative data, ward caseload (e.g. bed capacity), admission data (e.g. population characteristics), and number of VRErelated bacteremia cases.

\section{Conclusion}

VRE screening and isolation for patients at risk for colonisation in the general medicine ward can be considered a cost-effective infection prevention and control intervention in this simulation study. The intervention's cost-effectiveness varied depending on VRE prevalence and isolation effectiveness. This model would need to be adapted to more accurately estimate the impact in specific local contexts but can provide broad economic evidence to inform infection prevention and control practitioners, program planners and health policy decision-makers. 


\section{Supplementary information}

Supplementary information accompanies this paper at https://doi.org/10. 1186/s13756-019-0628-x

\section{Additional file 1. CHEERS Checklist.}

\section{Abbreviations}

AMR: Antimicrobial resistant; CADTH: Canadian Agency for Drugs and Technology for Health; CEAC: Cost-effectiveness acceptability curve; CET: Cost-effectiveness threshold; CHEERS: Consolidated Health Economic Evaluation Reporting Standards; Cl: Confidence interval; EVPI: Expected value of perfect information; ICER: Incremental cost-effectiveness ratio; ICU: Intensive care unit; IQR: Interquartile range; LOS: Length of stay; MRSA: Methicillin-resistant Staphylococcus aureus; PPE: Personal protective equipment; PSA: Probabilistic sensitivity analysis; QALY: Quality-adjusted life year; UTI: Urinary tract infection; VRE: Vancomycin-resistant enterococci

\section{Acknowledgements}

Not applicable.

\section{Authors' contributions}

SM developed the model, acquired the data, carried out analyses, interpreted the results, and drafted the manuscript. TF developed the model, acquired the data, and drafted the manuscript. JJ initiated the study, acquired the data and interpreted the results. BS initiated the study, interpreted the results, and supervised the study. All authors critically reviewed and revised the manuscript, and approved the final version of the manuscript.

\section{Funding}

This study did not receive any source of funding. Presentation of interim results at 2018 AMMI Canada - CACMID Annual Conference was supported by the Canadian Institute for Health Research (CIHR) Institute Community Support Travel Award (ISU-157466).

\section{Availability of data and materials}

All data generated or analysed during this study are included in this article.

\section{Ethics approval and consent to participate}

Not applicable.

\section{Consent for publication}

Not applicable.

\section{Competing interests}

The authors declare that they have no competing interests.

\section{Author details}

${ }^{1}$ Institute of Health Policy, Management and Evaluation, University of Toronto, 155 College Street, Suite 425, Toronto, ON M5T 3M6, Canada. ${ }^{2}$ Toronto Health Economics and Technology Assessment (THETA) Collaborative, University Health Network, 200 Elizabeth Street, 10th Floor, Room 247, Toronto, ON M5G 2C4, Canada. ${ }^{3}$ Dalla Lana School of Public Health, University of Toronto, 155 College Street, 6th Floor, Toronto, ON M5T 3M7, Canada. ${ }^{4}$ Department of Laboratory Medicine and Pathobiology, University of Toronto, 1 King's College Circle, Toronto, ON M5S 1A8, Canada. ${ }^{5}$ Public Health Ontario, 480 University Avenue, Suite 300, Toronto, ON M5G 1V2, Canada. ${ }^{6}$ ICES, G1 06, 2075 Bayview Avenue, Toronto, ON M4N 3M5, Canada.

Received: 1 August 2019 Accepted: 16 October 2019

Published online: 29 October 2019

\section{References}

1. Government of Canada. Canadian Antimicrobial Resistance Surveillance System 2017 Report [Internet]. 2017 [cited 2018 Apr 1]. Available from: https://www.canada.ca/en/public-health/services/publications/drugs-healthproducts/canadian-antimicrobial-resistance-surveillance-system-2017-reportexecutive-summary.html\#fn3
2. Johnstone J, Chen C, Rosella L, Adomako K, Policarpio ME, Lam F, et al. Patient- and hospital-level predictors of vancomycin-resistant Enterococcus (VRE) bacteremia in Ontario, Canada. Am J Infect Control. 2018;46(11):1266-71.

3. Lloyd-Smith P, Younger J, Lloyd-Smith E, Green H, Leung V, Romney MG. Economic analysis of vancomycin-resistant enterococci at a Canadian hospital: assessing attributable cost and length of stay. J Hosp Infect. 2013;85(1):54-9.

4. Siegel JD, Rhinehart E, Jackson M, Chiarello L, Gordon SM, Harrell L, et al. 2007 guideline for isolation precautions: preventing transmission of infectious agents in health care settings. Am J Infect Control. 2007;35:S65-164.

5. Cookson BD, Macrae MB, Barrett SP, Brown DFJ, Chadwick C, French GL, et al. Guidelines for the control of glycopeptide-resistant enterococci in hospitals *. J Hosp Infect. 2006;62:6-21.

6. Ontario Agency for Health Protection and Promotion PIDAC. Annex A: Screening, Testing and Surveillance for Antibiotic-Resistant Organisms (AROs) in All Health Care Settings. Toronto, ON; 2013.

7. Canadian Agency for Drugs and Technologies in Health. VancomycinResistant Enterococci Isolation and Screening Strategies: Clinical Evidence and Cost-Effectiveness. 2014.

8. Bodily M, McMullen KM, Russo AJ, Kittur ND, Hoppe-Bauer J, Warren DK. Discontinuation of reflex testing of stool samples for Vancomycin-resistant enterococci resulted in increased prevalence. Infect Control Hosp Epidemiol. 2013;34(08):838-40.

9. Muto CA, Giannetta ET, Durbin LJ, Simonton BM, Hew CLT, Farr BM. Costeffectiveness of perirectal Surevillance cultures for controlling Vancomycinresistant Enterococcus. Infect Control Hosp Epidemiol. 2002;23(8):429-35.

10. Grosse SD. Assessing cost-effectiveness in healthcare: history of the $\$ 50,000$ per QALY threshold. Expert Rev Pharmacoecon Outcomes Res. 2008; 8(2):165-78

11. Canadian Agency for Drugs and Technologies in Health (CADTH). Guidelines for the Economic Evaluation of Health Technologies: Canada. 4th ed. 2017. 1-76 p.

12. Anderson RM, May RM. Infectious diseases of humans: dynamics and control. Cambridge, U.K.: Oxford University Press; 1992.

13. Satilmis $L$, Vanhems $P$, Bénet $T$. Outbreaks of Vancomycin-resistant enterococci in hospital settings: a systematic review and calculation of the basic reproductive number. Infect Control Hosp Epidemiol. 2016; 37(3):289-94.

14. Williams V, Simor A, Kiss A, McGeer A, Hirji Z, Larios OE, et al. Is the prevalence of antibiotic-resistant organisms changing in Canadian hospitals? Comparison of point-prevalence survey results in 2010 and 2012. Clin Microbiol Infect. 2015;21:553-9.

15. Conly JM, Ofner-Agostini M, Paton S, Johnston L, Mulvey M, Kureishi A, et al. The emerging epidemiology of VRE in Canada: results of the CNISP passive reporting network, 1994 to 1998. Can J Infect Dis. 2001;12(6):364-70.

16. Stamper PD, Shulder S, Bekalo P, Manandhar D, Ross TL, Speser S, et al. Evaluation of BBL CHROMagar VanRE for detection of vancomycin-resistant enterococci in rectal swab specimens. J Clin Microbiol. 2010;48(11):4294-7.

17. Saunders L. Morbidity and Mortality Comparing Vancomycin-Sensitive E. faecium and Vancomycin-Resistant E. faeciu. Am J Infect Control [Internet] 2004 May 1 [cited 2019 Apr 6];32(3):E16-7. Available from: https:// linkinghub.elsevier.com/retrieve/pii/S0196655304001269

18. Daneman N, Rishu A, Xiong W, Palmay L, Fowler RA. Antimicrobial cost savings assocaited with shorter duration treatment for bloodstream infections. J Assoc Med Microbiol Infect Dis Canada. 2016;1(2):33-4.

19. Billington EO, Phang SH, Gregson DB, Pitout JDD, Ross T, Church DL, et al. Incidence, risk factors, and outcomes for Enterococcus spp. blood stream infections: a population-based study. Int J Infect Dis. 2014;26:76-82.

20. Linden PK, Pasculle AW, Manez R, Kramer DJ, Fung JJ, Pinna AD, et al. Differences in outcomes for patients with bacteremia due to vancomycinresistant Enterococcus faecium or vancomycin-susceptible E. faecium. Clin Infect Dis. 1996;22(4):663-70.

21. St. Joseph's Health Center. Types of Rooms [Internet]. [cited 2017 Dec 10] Available from: https://stjoestoronto.ca/patient-care-and-services/what-toexpect-as-a-patient/types-of-rooms

22. Nasr G, Canchi D, Large J, Martin S, Sandin R, Greene J. A hospital based VRE screening for patients with hematological malignancies: a cost benefit analysis. IDSA Poster Abstr. 2011.

23. Lee BY, Bailey RR, Smith KJ, Robert R, Strotmeyer ES, Lewis GJ, et al. Universal methicillin-resistant Syaphylococcus aureus (MRSA) surveillance for adults at hospital admission: an economic model and analysis. Infect Control Hosp Epidemiol. 2010;31(6):598-606. 
24. Haran MJ, Lee BB, King MT, Marial O, Stockler MR. Health status rated with the medical outcomes study 36-item short-form health survey after spinal cord injury. Arch Phys Med Rehabil. 2005;86(12):2290-5.

25. Tengs T, Wallace AMY. One thousand health-related quality-of-life estimates. Med Care. 2000;38(6):583-637.

26. Selai C, Rosser R. Eliciting EuroQol descriptive data and utility scale values from inpatients. Pharmacoeconomics. 1995;8(2):147-58.

27. Revicki D, Wood M. Patient-assigned health state utilities for depressionrelated outcomes: differences by depression severity and antidepressant medications. J Affect Disord. 1998;48:25-36.

28. Mittmann N, Trakas K, Risebrough N, Liu BA. Utility scores for chronic conditions in a community-dwelling population. Pharmacoeconomics. 1999; 15(4):369-76.

29. Statistics Canada. Life Tables, Canada, Provinces and Territories 1980/1982 to 2014/2016 [Internet]. 2018 [cited 2018 Nov 29]. Available from: https:// www150.statcan.gc.ca/n1/pub/84-537-x/84-537-x2018002-eng.htm

30. Torrance GW. Measurement of health state utilities for economic appraisal: A review. J Health Econ [Internet]. 1986 Mar 1 [cited 2019 Mar 6];5(1):1-30. Available from: https://www.sciencedirect.com/science/article/pii/01676296 86900202?via\%3Dihub

31. Public Health Agency of Canada. Vancomycin-Resistant Enterococci Infections in Canadian Acute-Care Hospitals. 2013.

32. Husereau D, Drummond M, Petrou S, Carswell C, Moher D, Greenberg D. Consolidated health economic evaluation reporting standards (CHEERS)-explanation and elaboration: a report of the ISPOR health economic evaluations publication guidelines task force. Value Health. 2013;16:231-50.

33. Ajao AO, Harris AD, Roghmann M-C, Johnson JK, Zhan M, McGregor JC, et al. Systematic review of measurement and adjustment for colonization pressure in studies of methicillin-resistant Staphylococcus aureus, vancomycin-resistant enterococci, and Clostridium difficile acquisition. Infect Control Hosp Epidemiol [Internet]. 2011 May [cited 2019 Jul 29];32(5):481-9. Available from: http://www.ncbi.nlm.nih.gov/pubmed/21515979.

34. Shadel BN, Puzniak LA, Gillespie KN, Lawrence SJ, Kollef M, Mundy LM. Surveillance for Vancomycin-resistant enterococci: type, rates, costs, and implications. Infect Control Hosp Epidemiol. 2006;27(10):1068-75.

35. Perencevich EN, Fisman DN, Lipsitch M, Harris AD, Morris JG Jr, Smith DL. Projected benefits of active surveillance for Vancomycin-resistant enterococci in intensive care units. Clin Infect Dis. 2004;38(8):1108-15.

36. Faron ML, BWB NAL. Resistance Mechanisms, Epidemiology, and Approaches to Screening. J Clin Microbiol. 2016;54(10):2436-47.

37. Montecalvo MA, Jarvis WR, Uman J, Shay DK, Petrullo C, Horowitz HW, et al. Costs and savings associated with infection control measures that reduced transmission of vancomycin-resistant enterococci in an endemic setting. Infect Control Hosp Epidemiol. 2001;22(7):437-42.

38. Hamel M, Zoutman D, O'Callaghan C. Exposure to hospital roommates as a risk factor for health care-associated infection. Am J Infect Control. 2010; 38(3):173-81.

39. Derde LPG, Cooper BS, Goossens H, Malhotra-Kumar S, Willems RJL, Gniadkowski $M$, et al. Interventions to reduce colonisation and transmission of antimicrobial-resistant bacteria in intensive care units: an interrupted time series study and cluster randomised trial. Lancet Infect Dis. 2014;14(1):31-9.

40. Huskins WC, Huckabee CM, O'Grady NP, Murray P, Kopetskie H, Zimmer L, et al. Intervention to reduce transmission of resistant Bacteria in intensive care. N Engl J Med. 2011;364(15):1407-18.

41. Lapointe-Shaw L, Voruganti T, Kohler P, Thein H-H, Sander B, McGeer A. Cost-effectiveness analysis of universal screening for carbapenemaseproducing Enterobacteriaceae in hospital inpatients. Eur J Clin Microbiol Infect Dis. 2017;36(6):1047-55.

42. Papia G, Louie M, Tralla A, Johnson C, Collins V, Simor AE. Screening highrisk patients for methicillin-resistant staphylococcus Aureus on admission to the hospital is it cost effective? Infect Control Hosp Epidemiol. 1999; 20(07):473-7.

\section{Publisher's Note}

Springer Nature remains neutral with regard to jurisdictional claims in published maps and institutional affiliations.

\section{Ready to submit your research? Choose BMC and benefit from:}

- fast, convenient online submission

- thorough peer review by experienced researchers in your field

- rapid publication on acceptance

- support for research data, including large and complex data types

- gold Open Access which fosters wider collaboration and increased citations

- maximum visibility for your research: over $100 \mathrm{M}$ website views per year

At $\mathrm{BMC}$, research is always in progress.

Learn more biomedcentral.com/submissions 